\title{
Políticas e práticas de saúde rumo à eqüidade
}

\author{
HEALTH POLICIES AND PRACTICES TOWARD EQUITY \\ POLÍTICAS Y PRÁCTICAS DE SALUD HACIA LA EQUIDAD
}

\author{
Roseni Rosângela de Sena', Kênia Lara Silva²
}

\section{RESUMO}

O texto abordou as desigualdades e a exclusão social em saúde e estabeleceu as suas inter-relações e determinações sociais, históricas, culturais e econômicas. Apontou as iniqüidades, como conseqüência das desigualdades na produção e na distribuição das riquezas expressas em serviços e bens materiais. Discutiu as práticas de gestão no campo da saúde, como favorecedoras de equiidade, indicando a gestão compartilhada, como uma ferramenta que potencializa os valores de cidadania e respeito à diversidade. Abordou a participação e o controle social como práticas que promovem eqüidade nos serviços de saúde. Comentou a experiência da Secretaria Municipal de Políticas Sociais e de Saúde de Belo Horizonte com o trabalho em territórios geopolíticos-sociais com práticas de gestão, assistência e controle social que contribuem para a eqüidade. Concluiu que, além das análises e macrointervenções, é fundamental tomar os espaços micro do trabalho para reflexão e atuação na construção da equidade.

\section{DESCRITORES}

Iniquiidade social.

Saúde pública.

Políticas Públicas de Saúde.

\begin{abstract}
The text approaches the inequalities and the social exclusion in health establishing its social, historical, cultural and economic inter-relations and determinations. It points to the iniquities as consequence of the inequalities in the production and distribution of the wealth expressed by access to services and material goods. It discusses the practices of management, in the field of the health, as supporters of the equity, indicating the shared management as a tool that promotes the values of citizenship and respect to the diversity. It approaches the participation and the social control as practices that promote equity in the health services. It comments the experience of the Department of Social Politics and Health of Belo Horizonte with the work in geopolitical and social territories with practices of management, assistance and social control that contribute for equity. It concludes that, beyond the analyses and macro interventions, it is basic to take the spaces micro of the work for reflection and performance in the construction of equity.
\end{abstract}

\section{KEY WORDS}

Social inequity.

Public health.

Health Public Policy .

\section{RESUMEN}

Este texto abordó las desigualdades y la exclusión social en salud, estableciendo sus interrelaciones y determinaciones sociales, históricas, culturales y económicas. Se señaló a las iniquidades como consecuencia de las desigualdades en la producción y distribución de las riquezas, expresadas en servicios y bienes materiales. Se discutió las prácticas de la gerencia, en el campo de la salud, como facilitadores de la equidad, indicando a la gerencia compartida como herramienta que potencialaza los valores de ciudadanía y respeto a la diversidad. Se abordó la participación y el control social como prácticas que promueven la equidad en los servicios de salud. Se comentó sobre la experiencia del Departamento $\mathrm{Mu}$ nicipal de Políticas Sociales y de Salud de Belo Horizonte sobre el trabajo en territorios geopolíticos y sociales, con prácticas de gerencia, asistencia y control social que contribuyeron para la equidad. Se concluyó que además de los análisis y de las macro-intervenciones, es fundamental tomar los espacios micro del trabajo para reflexionar y actuar en la construcción de la equidad.

\section{DESCRIPTORES}

Inequidad social.

Salud pública.

Políticas Públicas de Salud.

\author{
1 Enfermeira. Doutora \\ em Enfermagem. \\ Professora Emérita \\ da Universidade \\ Federal de Minas \\ Gerais. Pesquisadora \\ do CNPq. Coordena- \\ dora do Núcleo de \\ Estudos e Pesquisas \\ sobre Ensino e \\ Prática de Enferma- \\ gem (NUPEPE). Belo \\ Horizonte, MG, Brasil. \\ rosenisena@uol.com.br \\ 2 Enfermeira. Douto- \\ randa em Enferma- \\ gem. Professora da \\ Pontifícia Universi- \\ dade Católica de \\ Minas Gerais. \\ Pesquisadora do \\ NUPEPE. Belo \\ Horizonte, MG, Brasil. \\ kenialara17@ \\ yahoo.com.br
}




\section{INTRODUÇÃO}

O texto apresentado é fruto de reflexões das autoras, integrantes do Núcleo de Estudos e Pesquisa sobre o Ensino e a Prática da Enfermagem (NUPEPE) da Escola de Enfermagem da Universidade Federal de Minas Gerais, e traduz inquietações sobre as quais refletimos em nosso cotidiano como parte da nossa prática docente, assistencial e de produção do conhecimento.

As desigualdades sociais e sua relação com a saúde são objeto de estudo de diversos autores que têm se apropriado de diferentes abordagens teórico-metodológicas para explicar e analisar o impacto das condições de vida na saúde e da saúde nas condições de vida ${ }^{(1-6)}$. A expansão desses estudos, na América Latina, acompanha o crescimento da Epidemiologia Social e da Saúde Coletiva e tem permitido acumulações importantes na área.

No contexto da globalização e do neoliberalismo, surgem novas demandas para a análise das relações já conhecidas e de outras que agregam perspectivas ainda não exploradas no campo. É necessário avançar, por exemplo, na realização de estudos que demonstrem como as políticas e práticas de saúde respondem às desigualdades e iniqüidades; como a formação dos profissionais de saúde tem contribuído para a luta contra a iniqüidade e quais dispositivos podem ser utilizados para a reflexão e recons-trução das práticas de saúde que possam contribuir para a equidade $^{(7)}$. Optamos por encaminhar a discussão nesta direção.

Consideramos que a iniqüidade, ao se expressar no cotidiano da vida, torna-se uma determinante de nosso pensar, fazer e sentir em saúde, exigindo nosso envolvimento para a transformação desta realidade inaceitável.

Ponderamos que o cenário atual das desigualdades e iniqüidades em saúde no Brasil e América Latina está determinado por uma distribuição inadequada na produção e na distribuição das riquezas, gerando uma exclusão social e econômica, por conseguinte, pobreza e miséria. A distribuição desigual gera condições de vida que comprometem a saúde e a qualidade de vida de grandes setores da sociedade brasileira e latinoamericana. No presente texto, buscamos entender as implicações deste quadro no direcionamento das práticas de saúde. Pretendemos enfocar as práticas de gestão, assistência, participação e controle social na sua relação com a construção da equidade. Para tanto, tomamos o cenário da organização de políticas sociais no município de Belo Horizonte para exemplificar a materialidade de políticas e práticas que vêm sendo construídas.

\section{DESIGUALDADE, INIQÜIDADE E EXCLUSÃO SOCIAL E EM SAÚDE: INTER-RELAÇÕES E DETERMINAÇÕES}

As desigualdades no Brasil têm uma forte expressão regional. A região Sul é a mais desenvolvida em termos econômicos e sociais, convive com desigualdades expressas nas demais regiões. A região Nordeste sofre com as desigualdades que geram a pobreza e a miséria com índices que atingem $44 \%$ da população.

É importante destacar que as disparidades regionais no Brasil não se devem à escassez de recursos, mas à persistente iniquiidade. A região Nordeste é rica em recursos naturais e culturais que se expressam no modo de vida da população e são fontes de energia e de possibilidades para superar as condições adversas. Por isso, não podemos caracterizar a Região Nordeste como a mais pobre e sim como a mais desigual, com riquezas que não se expressam nos índices econômicos.

Muitas vezes expressões como desigualdade, iniqüidade e exclusão são utilizadas como termos sinônimos ou com significados muito próximos. Entretanto, como revelam resultados diferentes a respeito da produção, da distribuição e do acesso a bens e serviços em determinada sociedade, é preciso diferenciá-las.

Entende-se por desigualdade as diferenças produzidas pela inserção social dos indivíduos e que estão relacionadas com a repartição do poder e da propriedade ${ }^{(2)}$. A desigualdade só existe em relação(4) na qual iguais e desiguais podem ser comparados em função de uma situação comum. Já o conceito de iniqüidade remete a uma desigualdade evitável, desnecessária ou não justificável $^{(8)}$. Esse julgamento, ao tratar de questões como níveis de saúde, exige, necessariamente, o estabelecimento de uma linha divisória, tomada como parâmetro de justo ou injusto e orientada, em grande parte, por princípios morais, éticos e políticos, que são historicamente determinados e socialmente incorporados como parte dos valores de uma sociedade $^{(9)}$. Assim, é compreensível que desigualdades gerem iniqüidades.

Verificamos que a situação de diferenças econômicas e sociais entre grupos populacionais dentro do Brasil tem repercussões importantes na qualidade de vida e saúde das populações. Essa diferença determina as condições de vida e de saúde e requer decisão de como os recursos financeiros, tecnológicos e a força de trabalho em saúde serão utilizados para transformar este quadro.

A saúde caracteriza-se por diversidades regionais importantes, mas, de modo geral, o que se observa são amplos setores da população excluídos do acesso aos serviços de 
saúde. Para agravar o quadro, convivemos com a transição demográfica e epidemiológica, produzindo mudanças na estrutura etária da população e no perfil de adoecimento. Assim, tão importante quanto buscar a equidade, uma distribuição justa dos bens e dos serviços, é fundamental construir políticas que promovam a inclusão social, fundamental na afirmação da cidadania, uma vez que a inclusão é premissa para a eqüidade ${ }^{(7)}$.

É fundamental reconhecer que as condições de saúde e de adoecimento estão determinadas pelas desigualdades que se expressam em indicadores de morbi-mortalidade, no acesso e na distribuição dos serviços, na formulação das políticas e na construção das práticas de saúde. Optamos por trabalhar neste texto o enfoque das práticas de saúde e de como elas podem promover a eqüidade, superando as desigualdades, as iniqüidades e a exclusão social. Essa definição está determinada pelo lugar de onde exercemos o nosso papel de cidadãs na sociedade e, mais especificamente, como profissionais de saúde e do ensino.

\section{PRÁTICAS DE GESTÃO QUE PROMOVEM A EQÜIDADE}

A formulação de políticas atende interesses de setores da sociedade sendo que, em uma situação de desigualdade e de iniqüidade, apenas alguns estão representados nos espaços da formulação das políticas. Historicamente, convivemos com políticas de saúde que marcavam a segmentação da população de forma estratificada, com valorização da alta tecnologia e o desmantelamento das ações de atenção básica e saúde coletiva. A hegemonia dessas políticas teve repercussões importantes no custo e na qualidade do cuidado em saúde e nas práticas em saúde coletiva, com priorização da atenção médica, privatista, individual e curativa.

Ao definir pela possibilidade de reverter o quadro atual das desigualdades e iniqüidade, torna-se uma premissa incorporar os princípios da gestão compartilhada, ampliando a possibilidade da tomada de decisão com os trabalhadores e com segmentos da sociedade que têm a responsabilidade e podem contribuir para a formulação e a execução das políticas.

A gestão compartilhada é um pressuposto teórico-metodológico que incorpora os valores de democracia, cidadania e de respeito à diversidade. Considerando as desigualdades que se expressam na iniquiidade, é fundamental no setor saúde superar as modalidades burocráticas e centralizadas de gestão, para construir as formas de gestão que considerem a rede de cuidado de saúde como espaço de gestão em todos os seus âmbitos e instâncias de produção de serviços de saúde.
Entendemos a gestão compartilhada como uma possibilidade de que o poder seja exercido por todos: gestores, trabalhadores e usuários, com mecanismos de tomada de decisão e de execução do trabalho, a qual tem capilaridade e se expressa na capacidade de todas as pessoas envolvidas conhecerem as situações em que se quer intervir. Assim, criam-se espaços para decidir pelo mais oportuno, que atende demandas e necessidades e para garantir a eficiência das ações e sua sustentabilidade.

Os espaços de gestão tornam-se espaços de escuta e de expressão das diferentes vozes dos atores que se comprometem com a decisão, com os processos e com os resultados da intervenção. É necessário reconhecer que os atores ocupam lugares diferentes que os fazem expressar interesses e vontades que precisam ser pactuadas. Porém, é no confronto dessas diferenças e interesses que se pode construir as possibilidades de superação das diferentes formas em que se expressa a exclusão.

\section{DEMOCRACIA E SUPERAÇÃO DAS DESIGUALDADES: PARTICIPAÇÃOE CONTROLE SOCIAL NA PROMOÇÃO DA EQÜIDADE}

Entendemos que qualquer prática e política para equidade deve, primeiramente, promover inclusão social e afirmação da cidadania. A participação da sociedade de forma aberta e democrática contribui para este movimento, uma vez que representa, em si, o exercício de cidadania, de capacidade de regular e administrar o poder, de compartilhar os interesses e as responsabilidades.

$\mathrm{Na}$ história da saúde no Brasil esse exercício tem sido construído por forças que se expressaram no direito da participação e no controle social sobre as ações de saúde. Os Conselhos e as Conferências de Saúde, como espaços institucionais de participação da sociedade na formulação e execução das políticas de saúde, tem sido um fator indiscutível para o avanço das práticas de equidade no setor saúde. A capilaridade que esses aparatos assumem com a organização de conselhos locais, distritais, regionais, municipais, estaduais é um mecanismo de inclusão social ao permitir que os interesses de grupos marginalizados e excluídos sejam vocalizados.

Assim, mesmo reconhecendo todos os desafios e os obstáculos impostos para estas práticas, como a manipulação político-partidária, os jogos de interesses que reproduzem as formas de desigualdade, as lideranças que concentram poder, a inadequada capacitação para o exercício deste poder, a utilização dos espaços para defesa de interesses particulares ou de grupos específicos, deve-se reconhecer a possibilidade de o controle social constituir-se num mecanismo promotor de inclusão. 
A eqüidade no campo da saúde requer um compromisso com a transformação das relações políticas, econômicas, sociais e culturais. Essas possibilidades se constroem nas redes de solidariedade sustentadas em confiança e relações de vínculos. Os diversos movimentos da sociedade civil organizada traduzem anseios da população para a expressão da cidadania e da participação que representa uma conquista. Podemos citar como exemplo a rede de educação popular em saúde como experiência de cidadania compartilhada.

A sociedade brasileira ainda guarda resquícios dos períodos de governos autoritários, nos quais o exercício da cidadania é restrito a determinados grupos de expressão econômica e social que, ao se expressarem nas organizações de interesses por causas, propósitos ou tema, também reproduzem a exclusão.

Construir e expressar a força da sociedade civil ainda é um desafio a ser superado. Primeiro, porque a sociedade civil se organiza a partir de laços de confiança e de solidariedade que foram rompidos e estão em reconstrução. Segundo, porque a superação das desigualdades e da iniqüidade pressupõe

desenvolvimento de uma base econômica com dinâmica própria, uma densa sociedade civil sobre bases de equidade social, o livre acesso aos meios de comunicação, o controle da corrupção e do clientelismo na gestão pública $^{(10)}$.

Por último, é muito importante que o País se torne independente na formulação de seu modelo de desenvolvimento, incluindo as políticas sociais para estabelecer estratégias de superação das desigualdades. Assim, é fundamental estabelecer com setores da sociedade as premissas para que o desenvolvimento não se ancore somente nos indicadores econômicos que, no caso brasileiro e de outros países da América Latina, têm sido determinantes para a concentração da renda. É necessário incorporar, nas políticas de desenvolvimento, o capital social e humano.

\section{PRÁTICAS EQÜÂNIMES NO COTIDIANO DA ASSISTÊNCIA À SAÚDE: O ENFOQUE NO SUJEITO}

No cotidiano da assistência à saúde é revelada a face conhecida e a face oculta da desigualdade em toda a rede de serviços, expressa nas possibilidades de acesso, de resolubilidade e de uma assistência oportuna.

Não são suficientes os discursos de que o modelo por ser biológico, curativo e individual não dá conta de atender as demandas e necessidades da população. Por trás da formulação e de expressão das práticas em saúde existe também uma reprodução da exclusão, uma vez que estas concretizam uma visão do processo saúde-doença imbuída das desigualdades por raça, gênero e renda.
Quando incluímos um conceito ampliado de saúde, há a expressão da eqüidade por considerar a determinação social, cultural e econômica que remete a todas as formas de viver com produção e distribuição das riquezas. Porém, o conceito ainda não impregnou as práticas de novos valores, atitudes, habilidades e competências na gestão, na assistência e nos processos formativos.

As práticas equânimes no cotidiano da assistência à saúde revelam-se na concretização do processo de trabalho que se organiza com compartilhamento de poderes e de decisões entre os diferentes sujeitos: trabalhadores e usuários. É na micropolítica do trabalho em saúde que a negociação e o cuidado compartilhado são assumidos como elementos de uma prática que supera as desigualdades nas relações e a exclusão de sujeitos nas decisões sobre o trabalho e sobre a saúde. Construir a prática desta forma traz conflitos, limites e dificuldades, pois é hegemônico na saúde o trabalho fragmentado e hierarquizado com poucos espaços para os sujeitos expressarem sua capacidade de auto-governo num exercício de diálogo na produção e distribuição do trabalho em saúde.

É iniqüidade também quando excluímos os sujeitos/usuários das decisões sobre sua saúde. Vivemos num mundo onde se divulga a liberdade; no entanto, bloqueia-se sua concretização ao não permitir que os sujeitos possam fazer escolhas, seja por não ouvi-los ou por não criar oportunidades para que façam tais escolhas. Construir práticas equânimes na assistência à saúde, exige, então, repensar os sentidos e significados da autonomia das pessoas nos seus modos de vida, na elaboração de políticas e na criação de oportunidades para as escolhas em saúde.

\section{O TERRITÓRIO COMO EXPRESSÃO DE DIFERENÇAS: CONSIDERAÇÕES A PARTIR DO CENÁRIO BELO HORIZONTE}

Para exemplificar como a equidade pode se materializar em práticas de gestão, assistência e controle social, tomamos a experiência do município de Belo Horizonte. Não se trata da melhor ou mais exitosa experiência na construção da eqüidade, mas revela a intencionalidade e a concretude de políticas e práticas que têm sido construídas nesse sentido.

Belo Horizonte (BH), capital de Minas Gerais é a terceira maior cidade do Brasil. Hoje, conta com cerca de 2,3 milhões de habitantes. É pólo de uma região metropolitana dinâmica, que atrai um volume cada vez maior de investimentos. $\mathrm{Na}$ última década, como um dos avanços no processo de consolidação do SUS, merece destaque a efetiva municipalização do Sistema de Saúde em Belo Horizonte, um dos municípios que mais contribuiu para o avanço do processo de descentralização no País. Nesse processo, iniciou a construção de um modelo de Vigilância à Saúde com a formação dos Distritos Sanitários, definição de territórios de responsabilidades segundo riscos sociais e epidemiológicos ${ }^{(11)}$. 
O município está organizado em nove áreas administrativas, definidas por recortes geográfico e populacional, que contêm Distritos Sanitários como mecanismo de regionalização do Sistema de Saúde municipal. A Estratégia de Saúde da Família, denominada BH Vida: Saúde Integral foi escolhida como proposta de reorganização da atenção básica no município, implantada a partir de 2002. Atualmente, 509 Equipes de Saúde da Família (ESF) estão em atividade, atendendo a quase 1,5 milhão de pessoas ${ }^{(12)}$.

O espaço territorial das áreas das Equipes de Saúde da Família (ESF) é o locus operacional de ações, que tem como objetivo promover e conservar a saúde da população. Na implantação da Estratégia de Saúde da Família em Belo Horizonte, a composição das áreas das equipes levou em consideração a quantidade de habitantes e o mapa de exclusão social, orientando uma organização diferenciada dos recursos assistenciais. O Mapa da Exclusão Social de Belo Horizonte foi obtido a partir de cruzamentos entre três índices desenvolvidos pelo Município, todos georreferenciados: Vulnerabilidade Social, Vulnerabilidade à Saúde e Qualidade de Vida Urbana.

O Índice de Vulnerabilidade à Saúde é uma combinação de diferentes variáveis num indicador que busca resumir informações relevantes que traduzem as desigualdades intraurbanas, apontando áreas prioritárias para intervenção e alocação de recursos, favorecendo a proposição de ações intersetoriais $^{(12)}$. Os indicadores utilizados referem-se a saneamento básico, habitação, educação, renda e indicadores de saúde e classificam os setores censitários do município em risco baixo, médio, elevado ou risco muito elevado ${ }^{(a)}$.

Apesar das limitações que a classificação por riscos pode apresentar e das divergências sobre sua utilização ${ }^{(13)}$, é preciso considerar que a divisão territorial por risco e vulnerabilidade apresenta elementos que contribuem para a discussão da eqüidade.

- Primeiro, porque reconhece a desigualdade como um fator importante na organização das práticas $e$ direciona a ação política e de governo, que busca a formulação de políticas públicas tomando a cidade na sua expressão. Esta ação define recursos financeiros, distribuição dos equipamentos sociais, recursos humanos de modo a responder as desigualdades que estão distribuídas na cidade de forma muito heterogênea. A utilização de um sistema de informação espacializado que congrega informações em saúde, demográficas, socioeconômicas, ambientais, de infraestrutura urbana, de oferta de serviços e outras, permite identificar problemas, determinantes e grupos mais vulnerabilizados e prioritários para a adoção de políticas públicas.

(a) A classificação seguiu os critérios: risco baixo: setores com valores inferiores ao médio; risco médio: setores censitários que tinham valores do índice de vulnerabilidade à saúde com $1 / 2$ desvio padrão em torno da média; risco elevado: setores com valores acima do risco médio até o limite de 1 desvio padrão; risco muito elevado: setores com valores acima do risco elevado.
- Segundo, porque analisa como a desigualdade $e$ seus determinantes expressam-se no território. Essa análise exige a desagregação de dados capazes de identificar a expressão das desigualdades em cada aglomerado, ou bairro. É a dinâmica do micro-espaço. Nesta compreensão, a cidade é um espaço de diversos, que se organizam segundo as condições que se estabelecerem nos territórios. A estrutura urbana releva e reproduz as desigualdades no que concerne à distribuição do poder social na sociedade, entendido como a capacidade diferenciada dos grupos e classes em desenca-dear ações que lhes permitam disputar os recursos urbanos.

- E, por fim, porque define ações estratégicas para uma prática que constrói a eqüidade. Neste sentido, a intersetorialidade é assumida como uma premissa fundamental na construção da eqüidade. Com essa intencionalidade, a Prefeitura de Belo Horizonte criou, em 2002, o Programa BH Cidadania. A iniciativa propõe uma ação integrada e maciça, focada no território vulnerável, com atuação articulada das políticas sociais, a fim de conseguir mudanças concretas na realidade de vida de pessoas vulnerabilizadas. O conceito de grupos vulnerabilizados para o BH Cidadania está estreitamente relacionado ao fenômeno da exclusão social e, para atender a este fenômeno, o Programa se ergueu sobre os conceitos de descentralização, intersetorialidade, territorialidade e participação cidadã e desenvolve cinco ações: Transferência de renda, Inclusão Produtiva, Educação, Saúde e Socialidade com foco no grupo familiar ${ }^{(14)}$.

É importante reconhecer que, apesar das limitações e dos desafios presentes em uma prática que procura romper com a visão individualista e setorializada dos problemas sociais de saúde, o Programa BH Cidadania materializa uma importante prática política, de gestão, de assistência e de controle e participação social na construção da cidadania local e mundial. Ao propor a redução dos níveis de pobreza e privação das comunidades, busca é eqüidade de oportunidades em direção a uma cidade mais inclusiva.

\section{CONSIDERAÇÕES FINAIS}

A análise sobre a iniqüidade no País permite reconhecer que as desigualdades mantêm e reproduzem as condições indesejáveis de saúde de grande parcela da população. $\mathrm{O}$ enfrentamento desta situação deve considerar as intervenções no âmbito macro, com formulação de políticas e execução eficiente de suas ações na promoção da equiidade.

A enfermagem, como prática social, deve se organizar para estabelecer alianças para a construção de possibilidades de superação das desigualdades geradoras das iniqüidades. Neste sentido, é preciso reconhecer a complexidade do tema e as muitas implicações que tem na cotidianidade do cuidado. 
É muito o trabalho que temos a seguir. Temos como fortaleza uma organização sólida, a parceria com as Escolas, Faculdades, Universidades e com os serviços de saúde. Para fazer frente a um desafio tão complexo, procuramos intensi-

\section{REFERÊNCIAS}

1. Barata RB. Condições de vida e situação de saúde. Rio de Janeiro: ABRASCO; 1997.

2. Barata RB. Desigualdades sociais e saúde. In: Campos GWS, organizador. Tratado de saúde coletiva. São Paulo: Hucitec; 2006. p. $457-86$.

3. Raphael D. Public health responses to health inequalities. Can J Public Health. 1998;89(6): 380-1.

4. Minayo MCS. Condiciones de vida, desigualdad y salud a partir del caso brasileño. In: Briceño-León R, Minayo MCS, Coimbra Junior CEA, editores. Salud y equidad: una mirada desde las ciencias sociales. Rio de Janeiro: FIOCRUZ; 2000. p. 55-71.

5. Dachs JNW. Inequidades en salud: cómo estudiarlas. In: Restrepo H, Málaga H. Promoción de la salud: cómo construir vida saludable. Bogotá: Ed. Médica Internacional; 2001. p. 84-104.

6. Dachs JNW, Bambas A, Casas JA. Determinantes sociais e econômicos de desigualdades em saúde na América Latina e no Brasil. In: Minayo MCS, Coimbra Junior CEA, editores. Críticas e atuantes: ciências sociais e humanas em saúde na América Latina. Rio de Janeiro: FIOCRUZ; 2005. p. 333-56.

7. Sena RR, Silva KL, Seixas CT. Practices in community health toward equity: contributions of Brazilian nursing. ANS Adv Nurs Sci. 2007;30(4):343-52. ficar as alianças com setores da sociedade civil comprometidos com o cuidado como defesa da vida, com igualdade, equidade, inclusão, democracia e solidariedade.

8. Whitehead M. The concepts and principles of equity and health. Int J Health Serv. 1991; 22(3):429-45.

9. Silva JB, Barros MBA. Epidemiologia e desigualdade: notas sobre a teoria e a história. Rev Panam Salud Publica. 2002; 12 (6):375-83.

10. Corragio JL. Desenvolvimento humano e educação: o papel das ONGs Latinoamericanas na iniciativa de educação para todos. $2^{a}$ ed. São Paulo: Cortez; 1999.

11. Campos SR, organizador. Sistema Único de Saúde em Belo Horizonte: reescrevendo o público. São Paulo: Xamã; 1998.

12. Belo Horizonte. Secretaria Municipal de Saúde. Plano Municipal de Saúde de Belo Horizonte: 2005-2008 [texto na Internet]. Belo Horizonte; 2005. [citado 2007 jun. 10]. Disponível em: http://www.pbh.gov.br

13. Spink MJP. Trópicos do discurso sobre risco: risco-aventura como metáfora na modernidade tardia. Cad Saúde Pública. 2001;17(6):1277-311.

14. Belo Horizonte. Secretaria Municipal de Coordenação de Política Social. BH Cidadania. Belo Horizonte; 2003. 